\title{
L'aile Nord, L'aile Sud
}

\author{
Anne Dandurand
}

\section{Excerpt from "North Wing, South Wing" \\ (Translated by Robert Majzels)}

You pace the room, unable to decide to take a bath. The darkness affords some comfort. To the north, between the blinds, you contemplate the highway and the movement of a hundred headlights, allowing yourself to be hypnotized by the glimmering anaconda. To the east, nothing. Dead streets. To the south: the opposite wing of the hotel, where everything sleeps, with the exception of someone on the seventh floor. Perhaps a couple? Imagining all those lives beneath their leaden dreams, you lose yourself in vague images.... Old horse, are you asleep on your feet?

Suddenly, directly opposite your window, a pair of owl eyes appear between the slats of the blinds. All drowsiness evaporates. These are no bird's eyes, rather a reflection in binoculars' lenses. Instinctively, you retreat. You can't have been seen. It must be a man-why do you assume it's a man? it could just as easily be a woman... - someone even more alone than you. A passing stranger whose dark pleasure is to spy.... A rush of pity tinged with disgust grips you.

Destiny has served you up a miserable prey.... And yet you're smiling. What scheme are you hatching? With great care you move an armchair to face the south window, you pull the blinds delicately to a horizontal position. Now you turn up the lights, turn the to on to the music video channel, mime the hustle of someone coming in from a fruitful evening. A glance to confirm you are still being observed. Yes. To frustrate the watcher, you undress quickly just out of the line of sight and, on a sudden whim, you go off and take a quick shower. Your hair still wet, you slip slowly into the armchair, offering your nudity to the voracity of the unknown gaze. You cross your legs. Cast a dreamy eye over this first silken night of spring. You are a gift of languour. 
Vos livres seraient publiés depuis une décennie maintenant; de peine et de misère, vous auriez acquis une incertaine notoriété... C'est-à-dire qu'une ou deux fois par année vous vous retrouveriez en tournée de lectures ou de promotion: silhouette affaissée dans l'anonymat des gares d'autobus, vos bagages trop lourds à bout de bras, luttant contre l'endormissement, et craignant de rater le départ... Mais avec au cœur cette joie discrète: la liberté d'un voyage solitaire...

(Ô le lent ballet des horizons derrière la vitre: plaines en damier à perte de vue, grêles bosquets de bouleaux, sapinières énigmatiques, somptueuses pavanes dans la lumière des raisons...)

En cas de promotion, vous auriez envisagé ces marathons-onze villes en douze jours-avec fatalité... Vous auriez à la longue appris à ravaler votre amertume, à réagir aimablement aux nombreux, trop nombreux journalistes vous redemandant, une fois de plus, de « raconter l'histoire » (en révélant ainsi, sans vergogne, qu'ils n'ont pas lu votre livre)... Vous vous seriez forgé d'utiles réparties sur la relative longueur de vos cheveux, vous vous piqueriez de toujours porter une broche intrigante afin de fournir à ces cervelles mollassonnes matière à entrevue... De loin en loin, vous rencontreriez un chroniqueur sérieux, le plus souvent une femme, qui aura lu avec ferveur votre œuvrette; plus rarement encore, qui aura tout lu de vous; s'engage alors, dans ces minutes-là, une conversation fébrile, vous, en plein désarroi, et ce débit haché de votre voix, celui d'une mère dont on réchappe de justesse le dernier-né...

Autrement, le plaisir des tournées de lectures vous draine. Tous ces visages inconnus tendus vers vous... Vous lisez pendant une heure ou deux ou trois, et répondez aux questions de la dizaine ou de la centaine de personnes, en majorité des dames bien mises; toujours, dans chaque ville, au fond de la salle trop éclairée, s'installe un jeune homme en cuir noirjamais le même jeune homme-hirsute, mal rasé, à la lippe boudeuse... Toujours, les paupières closes, il semble boire vos mots pendant que vous lisez... Dans votre voix, un imperceptible frémissement?

Le punk s'éclipse toujours le premier. Impossible de le talonner: l'on vous entoure, les plus timides surtout, qui n'ont pas voulu vous interroger devant tout le monde... Bon prince, vous les invitez à vous accompagner au bar de votre hôtel, pour une bière — $c^{\prime}$ est tout ce que vous avez les moyens de vous payer —et déjà, avant d'avoir trempé les lèvres dans votre verre, la tête vous danse: cette griserie de savoir vos livres aimés... Pour camoufler votre émoi, vous vous renseignez sur vos lecteurs: 1'une est ichtyologiste, l'autre secrétaire, les encadrent un cuisinier de cafétaria et un prof de philo à la retraite, et au bout de la table, deux copains dans la 
trentaine, peu diserts, médecins de campagne... Ceux-là, ou d'autres: ces visages, vers vous...

Intérieurement, l'imposture vous balafre: si vous vous souvenez avoir travaillé longtemps chacune de vos pages, chacune de vos phrases, vous n'ignorez pas que c'est en aveugle que vous avez trimé, l'oreille tendue vers... quoi? une musicalité?... ne vous appartenant pas... ineffable... En vous, le tâcheron ne se sentira jamais digne d'être aimé.

Les voix s'empâtent, les gestes s'écachent, la nuit va nous séparer; vous, la tête creuse, le pas pesant, vous regagnez votre chambre. Avec le temps, un rituel s'est élaboré: remonter le chauffage jusqu'à Tropique; si cela se peut, entrouvrir une fenêtre; verser une goutte d'eau de toilette sur l'ampoule, qu'une odeur familière maquille la pièce trop nue; sur le lit si vaste, disposer le quotidien local, le magazine littéraire du mois, vos volumes de Pascal, Rushdie et Bianciotti, puis le cahier à couverture rigide vous servant de journal. Autrefois, à portée de main, vous auriez aussi déposé la plume sur la tablette de papier ivoire. Afin, aux petites heures, d'écrire une lettre. Mais l'autre a cessé de correspondre avec vous. Le silence en est redouble.

Une nuit... Où donc était-ce? Pas dans une petite ville: vous entendez encore le flux, le reflux du trafic nocturne. Était-ce dans la capitale provinciale ou fédérale, ou dans la ville-reine? La fatigue a brouillé la géographie de votre mémoire.

Comme une mauvaise cantatrice qui n'en finirait plus d'agoniser sur scène, cet hiver-là avait trop longtemps poussé ses stridences. Mais dans la journée, un vent, une soierie de vent avait réchauffé cette cité dont vous avez oublié le nom. Tout souriait de soulagement, tout. Vous-même, pour une fois, y aviez perçu un espoir oblique.

On vous avait réservé une chambre dans l'aile nord d'un hôtel. Un peu ivre en revenant du bar, vous aviez failli vous tromper de direction. Après trois essais, vous aviez enfin introduit du bon côté la carte perforée dans la fente de la serrure, où un diode vert clignota: huit heures d'isolement vous ouvraient les bras. Vous aviez enlevé vos chaussures, entrebaîllé les fenêtres au nord, à l'est, au sud. L'air eut la douceur d'une paume. La radio grésillait trop pour que vous puissiez apprécier la musique. Avec la commande à distance de la télé, vous aviez constaté que la quarantaine de chaines ne déversaient que violences, balourdises ou mercantilisme effrénés. Vous aviez tout éteint. Sans vous dévêtir, vous vous allongez dans le noir. Votre cœur comme une yole ballottée entre l'épuisement et l'exaltation.

Cette première nuit du printemps vous appelle, vous tire, vous pourriez, rouge de gêne, mendier au chasseur de l'hôtel des adresses de cafés 
borgnes, afin d'y revoir, peut-être, votre insaisissable punk. Vous ne parvenez pas à vous y résoudre. Inutile de lire: les lignes imprimées tressauteraient. Des impatiences dans les jambes excluent le moindre assoupissement. Vous soupirez. Grillez une cigarette. Le corps en rade. Mais attiré par le large.

Vous arpentez la chambre sans vous décider à prendre un bain. La noirceur a pour vous des sollicitudes. Au nord, entre les lamelles du store, vous contemplez l'autoroute et la mouvance des centaines de phares, vous vous laissez hypnotiser par cet anaconda de lueurs. À l'est rien. Des rues mortes. Au sud: l'aile opposée de l'hôtel, où tout sommeille, hormis quelqu'un, au septième. Peut-être un couple? À imaginer toutes ces vies palpitant sous le plomb de leurs songes, vous vous perdez en des images imprécises... Vieux cheval, dormez-vous debout?

Soudain, exactement en face de votre fenêtre, deux yeux de hibou s'intercalent entre les lamelles du store. Toute somnolence s'évanouit. Ce ne sont pas des prunelles d'oiseau mais un reflet dans des lentilles de longues-vues. D'instinct vous vous écartez. L'on n'a pas pu vous repérer. Voilà un homme-pourquoi avez-vous présumé un homme? une femme, tout aussi bien... - un être plus seul encore que vous. Un individu de passage dont le noir plaisir est d'épier... Une pitié salie de dégoût vous empoigne.

Le destin vous a jeté en pâture une misérable proie... Mais vous souriez. Quelle manigance germe en vous? Avec un luxe de précautions, vous déménagez un fauteuil devant cette fenêtre vers l'aile sud, puis vous en actionnez le store avec délicatesse de manière à ce que les lamelles soient ouvertes. Ensuite, vous éclairez à giorno, allumez la télé à la chaîne des clips, mimez l'entrain de qui rentre d'une soirée fructueuse. D'un coup d'œil vous vérifiez si l'autre vous lorgne toujours. Oui. Pour l'agacer, vous vous dévêtez en vitesse juste en dehors de son champ de vision, et, par pure fantaisie, allez à la hâte vous doucher. Les cheveux encore mouillés, vous vous asseyez dans le fauteuil au ralenti, votre nudité offerte à la voracité du regard sans nom. Vous croisez les jambes. Semblez rêvasser dans l'air si soyeux de cette première nuit de printemps. Vous êtes un cadeau de longueur.

L'une à l'autre les minutes se maillent, vibrante dentelle. Entre vos cils, vous ne relâchez pas le guet. Avec une nonchalance des plus étudiées, votre bras glisse de l'accoudoir, votre main choit sur votre cuisse, très près de votre sexe. Sous vos doigts, le souvenir d'une autre cuisse, d'un autre sexe se superpose, impérieux. Vous fermez les yeux, l'âme inondée par des brisants... 
Était-ce en Angleterre? Dans un autobus, certainement, vous sentez dans vos reins son roulement obsédant, il est interdit de fumer, devant vous on jase en turc, en espagnol ou en tagal, une heure auparavant un arc-en-ciel par-dessus les champs bombait le dos, maintenant des relents de brandy, de sueur, de bergamote et de santal ondoient dans la demiobscurité, à côté de vous cet homme encore jeune, un échalas d'Allemand aux cheveux en brosse, aux lèvres qu' on voudrait ravager de baisers, vous avez mal contenu votre allégresse il y a deux heures lorsqu'il a plié sa grande carcasse dans le siège voisin du vôtre, depuis votre arrivée à ce colloque il y a trois jours, vous êtes sous le charme de cet étranger, de son humour un peu rosse, de sa voix moelleuse, parfois, lorsqu'il ne se croit pas observé, une moue fugace lui crispe la bouche, une peine pas si récente peut-être, un buisson d'orties au cœur peut-être, vous n'aviez pas osé lui parler, mais ce sourire de gamin qu'il vous a décoché en vous demandant s'il pouvait se mettre là, dans le siège voisin du vôtre, et ensuite cet échange à mi-voix entre son anglais cassé et le vôtre, hésitant, cette intimité si surprenante, et soudain ce silence, si étonnamment douillet, vous devez rafraîchir votre front, votre joue contre la vitre embuée, il le faut, qui êtes-vous alors: une quadragénaire aux espoirs broyés? un homosexuel toujours au secret? l'un ou l'autre absolument dans l'impuissance de dire votre désir, mais soit la désespérance, soit le diable vous mord, et avec une nonchalance des plus étudiées, votre bras glisse, votre main choit sur sa cuisse, très près de son sexe, et l'éternité semble vous envelopper dans sa cape, sous vos cils vous vous délectez de l'érection de l'Allemand, qui, tête renversée contre le haut du dossier, sourit, et votre main toujours immobile sur sa cuisse, et cet embrasement de votre sang...

Durant des mois par la suite vous évoqueriez ce sourire, ce qui précipite votre jouissance, comme maintenant derrière cette fenêtre d'hôtel dans une ville dont vous oublierez le nom. Depuis combien d'années ne connaissez-vous du plaisir que son ambiguïté?

La poitrine écrasée par la honte et la confusion, vous avez mal dormi. Ou trop peu. Avez-vous eu un cauchemar? Au loin, les Serbes bombardent toujours Sarajevo. Il crachine, l'aube s'enlise. Vous ramassez vos affaires, vous enfuyez. Personne dans le hall hormis le réceptionniste. À la gare, l'âcreté du café vous réconforte, peut-être. Autour de vous patiente une poignée de grands-mères proprettes. Après une première bouffée de cigarette, vous feuilletez le Bianciotti, et lorsque, page cinquante-cinq, vous lisez : « (...) la mémoire ne cessera de me dérouter, qui réserve aux faits un sort sans commune mesure avec leur importance (...) ", les larmes vous montent aux yeux. Vous sortirez-vous un jour des tristes labyrinthes où le désir se muselle?... 